\title{
TITLE:
}

\section{Asymmetric travelling waves in a square duct}

\author{
$\operatorname{AUTHOR}(\mathrm{S})$ :
}

Okino, Shinya; Nagata, Masato

\section{CITATION:}

Okino, Shinya ... [et al]. Asymmetric travelling waves in a square duct. JOURNAL OF FLUID MECHANICS 2012, 693: 57-68

ISSUE DATE:

2012-01-06

URL:

http://hdl.handle.net/2433/171946

RIGHT:

(C) Cambridge University Press 2012 


\section{Journal of Fluid Mechanics}

http://journals.cambridge.org/FLM

Additional services for Journal of Fluid Mechanics:

Journal of

Fluid Mechanics

Email alerts: $\underline{\text { Click here }}$

Subscriptions: Click here

Commercial reprints: Click here

Terms of use : $\underline{\text { Click here }}$

\section{Asymmetric travelling waves in a square duct}

Shinya Okino and Masato Nagata

Journal of Fluid Mechanics / Volume 693 / February 2012, pp 57 - 68

DOI: 10.1017/jfm.2011.455, Published online: 06 January 2012

Link to this article: http://journals.cambridge.org/abstract S0022112011004551

How to cite this article:

Shinya Okino and Masato Nagata (2012). Asymmetric travelling waves in a square duct. Journal of Fluid Mechanics,693, pp 57-68 doi:10.1017/jfm.2011.455

Request Permissions : $\underline{\text { Click here }}$ 


\title{
Asymmetric travelling waves in a square duct
}

\author{
Shinya Okino and Masato Nagata $\dagger$ \\ Department of Aeronautics and Astronautics, Graduate School of Engineering, Kyoto University, \\ Kyoto, 606-8501, Japan
}

(Received 11 March 2011; revised 3 October 2011; accepted 17 October 2011; first published online 6 January 2012)

Two types of asymmetric solutions are found numerically in square-duct flow. They emerge through a symmetry-breaking bifurcation from the mirror-symmetric solutions discovered by Okino et al. (J. Fluid Mech., vol. 657, 2010, pp. 413-429). One of them is characterized by a pair of streamwise vortices and a low-speed streak localized near one of the sidewalls and retains the shift-and-reflect symmetry. The bifurcation nature as well as the flow structure of the solution show striking resemblance to those of the asymmetric solution in pipe flow found by Pringle \& Kerswell (Phys. Rev. Lett., vol. 99, 2007, A074502), despite the geometrical difference between their cross-sections. The solution seems to be embedded in the edge state of square-duct flow identified by Biau \& Bottaro (Phil. Trans. R. Soc. Lond. A, vol. 367, 2009, pp. 529-544). The other solution deviates slightly from the mirror-symmetric solution from which it bifurcates: the shiftand-rotate symmetry is retained but the mirror symmetry is broken.

Key words: bifurcation, nonlinear instability, transition to turbulence

\section{Introduction}

Incompressible viscous flow through a straight duct with a square cross-section driven by a constant pressure gradient is believed to be linearly stable as in the case of pipe flow (Davey \& Drazin 1969; Tatsumi \& Yoshimura 1990). Therefore, there exists no solution which bifurcates from the laminar state and the transition to early stages of turbulence is abruptly caused by a finite-amplitude disturbance. The transitional regime for these flows is considered to be represented by the trajectory in the phase space wandering around unstable nonlinear solutions, called ECS (exact coherent structures), such as steady states, travelling waves and periodic solutions, which are disconnected from the laminar state. Hence, identifying ECS is important in understanding the turbulent dynamics and the laminar-turbulent transition from a dynamical-systems point of view. In pipe flow a number of ECS have been computed (Faisst \& Eckhardt 2003; Wedin \& Kerswell 2004; Pringle \& Kerswell 2007; Duguet, Pringle \& Kerswell 2008a; Duguet, Willis \& Kerswell 2008b; Pringle, Duguet \& Kerswell 2009). In contrast, only three travelling waves that are ECS have been reported so far in square-duct flow (Wedin, Bottaro \& Nagata 2009; Okino et al. 2010; Uhlmann, Kawahara \& Pinelli 2010). This is partially because solutions in a pipe are easily generalized to larger families by the rotational symmetry with an arbitrary angle, 
whereas those in a square duct only have $90^{\circ}$ rotational symmetry. The nonlinear travelling wave in a square duct in Wedin et al. (2009) was obtained for the first time by the self-sustaining process approach (Wedin et al. 2008), which was originally introduced by Waleffe (1997) for plane Couette flow. The solution has a four-vortex mean flow. However, the sense of the rotation of the vortices is opposite to the pattern observed at the transitional Reynolds number by a direct numerical simluations (DNS) study (Uhlmann et al. 2007). The mean flow of the solution in Uhlmann et al. (2010) shows a diagonally symmetric eight-vortex pattern. It is consistent with Prandtl's secondary flow of the second kind in square-duct turbulence. In Okino et al. (2010), a nonlinear analysis of internally heated square-duct flow is performed, extending the linear stability analysis of Uhlmann \& Nagata (2006). The isothermal solution is reached by a continuation approach in the parameter space. The dominant vortex structure of the mean flow of the solution resembles that of marginally turbulent flow (Uhlmann et al. 2007).

Investigation by DNS is another approach to understanding the transitional turbulent dynamics. Recently, much attention has been paid to the so-called edge state, which is the invariant set on the boundary between the laminar and turbulent states in phase space (Itano \& Toh 2001; Schneider, Eckhardt \& Yorke 2007; Duguet et al. 2008b; Schneider et al. 2008; Biau \& Bottaro 2009; Mellibovsky \& Meseguer 2009; Schneider \& Eckhardt 2009). The trajectory, which starts with an initial condition near the laminar-turbulent boundary, transiently approaches the edge state before the flow relaminarizes or evolves into turbulence.

For more thorough accounts of studies on square-duct flow readers should refer to the introduction of Okino et al. (2010).

In this paper, we are concerned with the mathematical, rather than physical, aspects of the transition to early stages of turbulence in a square-duct flow. We investigate the linear stability of the travelling wave solution found in Okino et al. (2010). Perturbations are split into four different symmetry groups. After having identified the linear critical points, we calculate bifurcating nonlinear solutions. We shall show evidence that one of the nonlinear solutions obtained matches with the edge state adequately.

\section{Linear stability analysis of the mirror-symmetric solution}

We take the Cartesian coordinates $\left(x_{*}, y_{*}, z_{*}\right)$ with the origin at the centre of the duct, where the $x_{*}$-axis is directed along the duct and the $y_{*^{-}}$and $z_{*}$-axes are parallel to the sides of the square cross-section. The subscript $*$ denotes dimensional variables. A pressure gradient is applied along the duct. The motion of the fluid in the duct is governed by the continuity equation, the Navier-Stokes equation and the no-slip boundary condition. The governing equations are non-dimensionalized by the length scale $b_{*}$, the time scale $b_{*}^{2} / v_{*}$ and the velocity scale $v_{*} / b_{*}$, where $b_{*}$ is half the side of the square cross-section of the duct and $\nu_{*}$ is the kinematic viscosity of the fluid. The flow is characterized by the Reynolds number, $R e=U_{c *} b_{*} / \nu_{*}$, where $U_{c *}$ is the centreline velocity of the laminar flow. The pressure drop has a proportionality with the centreline velocity: $\chi=3.3935 R e$, where $\chi$ is the non-dimensional pressure drop (Tatsumi \& Yoshimura 1990).

Periodicity in the $x$-direction with wavenumber $\alpha$ is imposed. We assume a travelling wave as a nonlinear solution: the velocity $\boldsymbol{u}=(u, v, w)$ is represented by $\boldsymbol{u}(x-c t, y, z)$, where $c$ is the phase velocity. 
The travelling wave found in Okino et al. (2010), which we refer to as ONWB hereafter, is invariant under the following transformations: shift-and-reflect,

$$
S:(u, v, w)(\xi, y, z) \rightarrow(u,-v, w)(\xi+\pi / \alpha,-y, z),
$$

and mirror-reflect,

$$
Z:(u, v, w)(\xi, y, z) \rightarrow(u, v,-w)(\xi, y,-z),
$$

where $\xi=x-c t$. The combination of $\boldsymbol{S}$ and $\boldsymbol{Z}$ leads to the shift-and-rotate,

$$
\boldsymbol{\Omega}:(u, v, w)(\xi, y, z) \rightarrow(u,-v,-w)(\xi+\pi / \alpha,-y,-z) .
$$

We follow the method of Okino et al. (2010) to investigate the linear stability of solution ONWB. Based on Floquet theory, the velocity perturbations, $\tilde{\boldsymbol{u}}$, are expanded as a Fourier series in the streamwise direction as follows:

$$
\tilde{\boldsymbol{u}}(x, y, z, t)=\sum_{l=-L}^{L} \tilde{\boldsymbol{u}}_{l}(y, z) \exp [\mathrm{i} l \alpha(x-c t)+\sigma t] .
$$

The Fourier spectra, $\tilde{\boldsymbol{u}}_{l}=\left(\tilde{u}_{l}, \tilde{v}_{l}, \tilde{w}_{l}\right)$, are expanded by modified Chebyshev polynomials, which satisfy the no-slip boundary conditions. The stream function $\tilde{\varphi}_{0}$ represents the cross-sectional flow, introduced to take the place of the mean parts, $\tilde{v}_{0}$ and $\tilde{w}_{0}: \tilde{v}_{0}=\partial_{z} \tilde{\varphi}_{0}$ and $\tilde{w}_{0}=-\partial_{y} \tilde{\varphi}_{0}$. The discretization of the equations is done by the Galerkin method. Examining the governing equations for the perturbations (see (4.4), (4.5), (4.8) and (4.9) of Okino et al. 2010), we find that the following four symmetry groups for $\tilde{v}_{l}, \tilde{w}_{l}, \tilde{u}_{0}$ and $\tilde{\varphi}_{0}$ are admissible:

symmetry IA :

$$
\tilde{v}_{l}\left\{\begin{array}{l}
\left(l^{+} ; e, e\right) \\
\left(l^{++} ; o, e\right)
\end{array}, \tilde{w}_{l}\left\{\begin{array}{l}
\left(l^{+} ; o, o\right) \\
\left(l^{++} ; e, o\right)
\end{array}, \tilde{u}_{0}(e, e), \tilde{\varphi}_{0}(o, o),\right.\right.
$$

symmetry IB:

$$
\tilde{v}_{l}\left\{\begin{array}{l}
\left(l^{+} ; e, o\right) \\
\left(l^{++} ; o, o\right)
\end{array}, \tilde{w}_{l}\left\{\begin{array}{l}
\left(l^{+} ; o, e\right) \\
\left(l^{++} ; e, e\right)
\end{array}, \tilde{u}_{0}(e, o), \tilde{\varphi}_{0}(o, e),\right.\right.
$$

symmetry IC :

$$
\tilde{v}_{l}\left\{\begin{array}{l}
\left(l^{+} ; o, e\right) \\
\left(l^{++} ; e, e\right)
\end{array}, \tilde{w}_{l}\left\{\begin{array}{l}
\left(l^{+} ; e, o\right) \\
\left(l^{++} ; o, o\right)
\end{array}, \tilde{u}_{0}(o, e), \tilde{\varphi}_{0}(e, o),\right.\right.
$$

symmetry ID:

$$
\tilde{v}_{l}\left\{\begin{array}{l}
\left(l^{+} ; o, o\right) \\
\left(l^{++} ; e, o\right)
\end{array}, \tilde{w}_{l}\left\{\begin{array}{l}
\left(l^{+} ; e, e\right) \\
\left(l^{++} ; o, e\right)
\end{array}, \tilde{u}_{0}(o, o), \tilde{\varphi}_{0}(e, e) .\right.\right.
$$

Here, $l^{+}$and $l^{++}$denote the odd and even Fourier mode in (2.4), respectively. The notation $e$ or $o$ indicates that the variable is an even or odd function with respect to the $y$-or $z$-coordinate. Symmetry IA is the same as the one that the solution ONWB possesses: the perturbations are invariant under the transformations $\boldsymbol{S}$ and $\boldsymbol{Z}$ (and therefore also under $\boldsymbol{\Omega}$ ). It is immediately verified that symmetries IB, IC and ID individually have symmetry $S, \boldsymbol{Z}$ and $\Omega$, respectively. The stability analysis for symmetry IA has already been reported in Okino et al. (2010). 

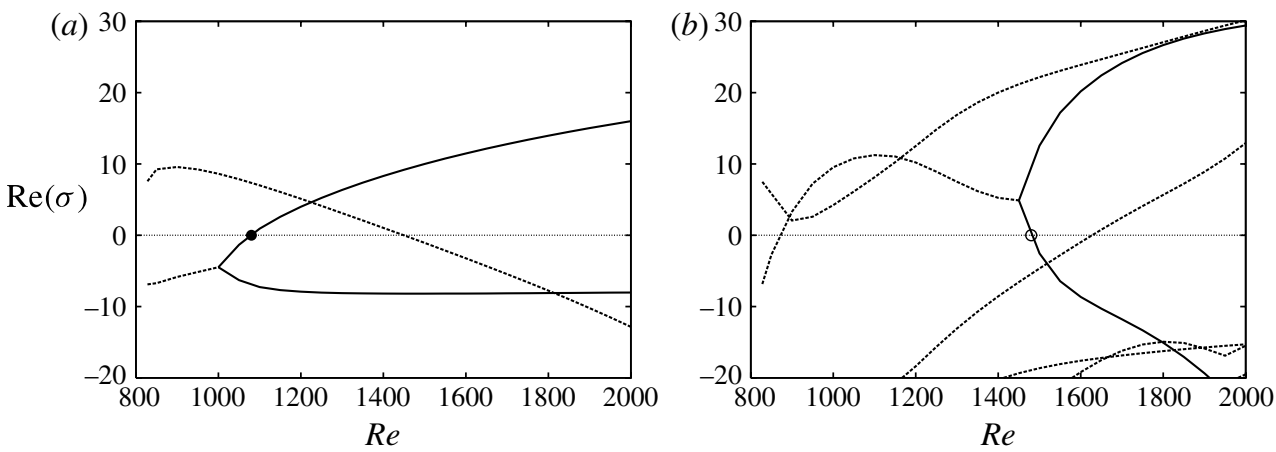

FIGURE 1. The real part of the growth rate, $\operatorname{Re}(\sigma)$, of the perturbations with symmetry IB on the $(a)$ lower and $(b)$ upper branches of ONWB with $\alpha=1.14$. Solid and dashed curves indicate that the growth rates are real and complex conjugate, respectively.
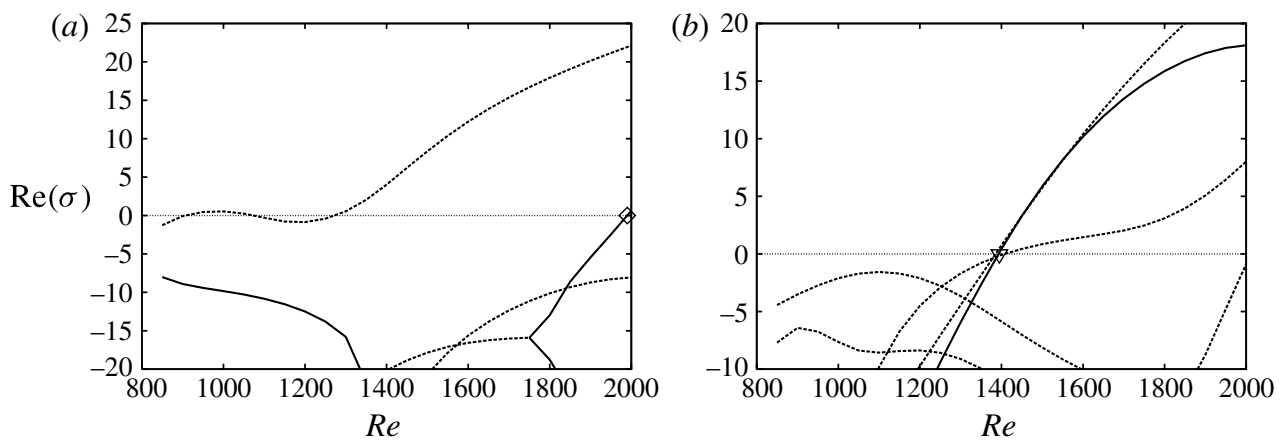

FIGURE 2. Same as figure 1, but for the perturbations with (a) symmetry IC and $(b)$ symmetry ID on the upper branch.

Figure 1 shows the real part of the growth rate $\sigma$ of the perturbations with symmetry IB on the lower and upper branches of ONWB. We choose $\alpha=1.14$ for which the bulk Reynolds number, $R e_{b}$, takes its minimum. $R e_{b}$ can be calculated by integrating the mean velocity over the cross-section (see (3.9) of Okino et al. 2010). As shown in figure $1(a)$, the largest growth rate has a complex-conjugate eigenvalue at $R e=827.5$ (the saddle-node of ONWB). It decreases as the Reynolds number increases. The neutral point is found at $R e \sim 1450$. The second largest eigenvalue, which starts as a complex-conjugate pair, splits into two real eigenvalues at $\operatorname{Re} \sim 1000$, and the larger one crosses zero at $R e \sim 1080$ (filled circle). No other neutral points are found on the lower branch within the parameter range of our investigation $(R e \leqslant 3000)$ when perturbations with symmetries IA, IC and ID are considered.

Figure 1(b) shows that the second eigenmode with symmetry IB on the upper branch, associated with a complex eigenvalue at the saddle-node of ONWB, crosses zero at $R e \sim 880$ and splits into two real modes at $R e \sim 1450$. After the split the lower branch mode becomes neutral when $R e \sim 1480$ (open circle). As shown in the figure $12(b)$ of Okino et al. (2010), all the neutral modes with symmetry IA on the upper branch are associated with a complex-conjugate pair. The growth rate of the perturbations with symmetry IC on the upper branch is shown in figure 2(a). The leading eigenvalue appears as a complex-conjugate pair. They cross zero three times 

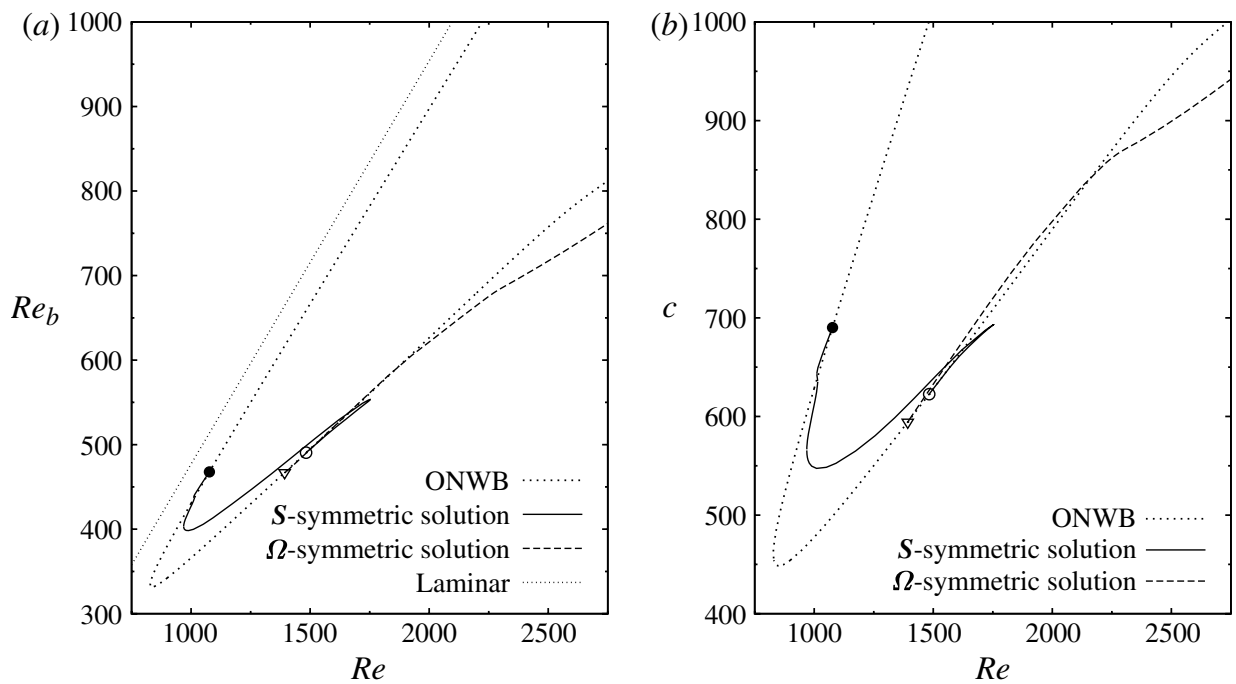

FIgURE 3. (a) The bulk Reynolds number versus the Reynolds number and $(b)$ the phase velocity versus the Reynolds number for $\alpha=1.14$. Dotted curve: ONWB. Solid curve: the $S$-symmetric solution. Dashed curve: the $\Omega$-symmetric solution. Thin dotted line: the laminar state, $R e_{b}=0.47704 R e$. The circles and the inverted triangle show the bifurcation points.

before $R e \sim 1300$ and take positive growth rates thereafter. Only one neutral mode associated with a real eigenvalue is found for $R e \sim 1990$ (open diamond). Figure 2(b) shows that the upper branch solution is stable to perturbations with symmetry ID before five unstable eigenmodes (one real and two complex conjugate pairs) appear almost together near $R e=1400$.

\section{Bifurcation of asymmetric travelling wave solutions}

We are interested only in nonlinear solutions that bifurcate from ONWB at the neutral points with real eigenvalues, as seen in figures 1 and 2. Using the eigenfuction at the neutral points as an initial guess for the Newton-Raphson method, we obtain two bifurcating solutions from ONWB with $\alpha=1.14$. The bulk Reynolds numbers, $R e_{b}$, and phase velocities, $c$, for these solutions as well as ONWB are plotted against the Reynolds number in figure 3. Also, the skin friction, $\lambda$, versus the bulk Reynolds number is shown in figure 4. Here, we define the skin friction, $\lambda=4 \chi / R e_{b}^{2}(\chi$ is the non-dimensional pressure drop in the streamwise direction), as a measure of the nonlinearity. The dotted curve in figures 3 and 4 indicates the ONWB branch. A new solution, which results from the instability due to symmetry IB and retains only symmetry $S$, bifurcates subcritically from the lower branch of ONWB at $R e=1077, R_{b}=468$ (filled circle), undergoes two turning points at $R e=969$ and 1753 , and terminates on the upper branch of ONWB at $R e=1483, R e_{b}=490$ (open circle). This solution branch connects the bifurcation points indicated by the filled and open circles in figure 1. Another new solution, which results from the instability due to symmetry ID and possesses only symmetry $\Omega$, bifurcates supercritically from the upper branch of ONWB at $R e=1393, R e_{b}=467$ (open inverted triangle in figure $3 a$ and also in figure $2 b$ ). Comparison of figure $3(a)$ and figure $3(b)$ shows that the phase velocity is $30 \sim 50 \%$ larger than the mean velocity for both ONWB and the two asymmetric solutions at fixed $R e$. We have used the truncation 


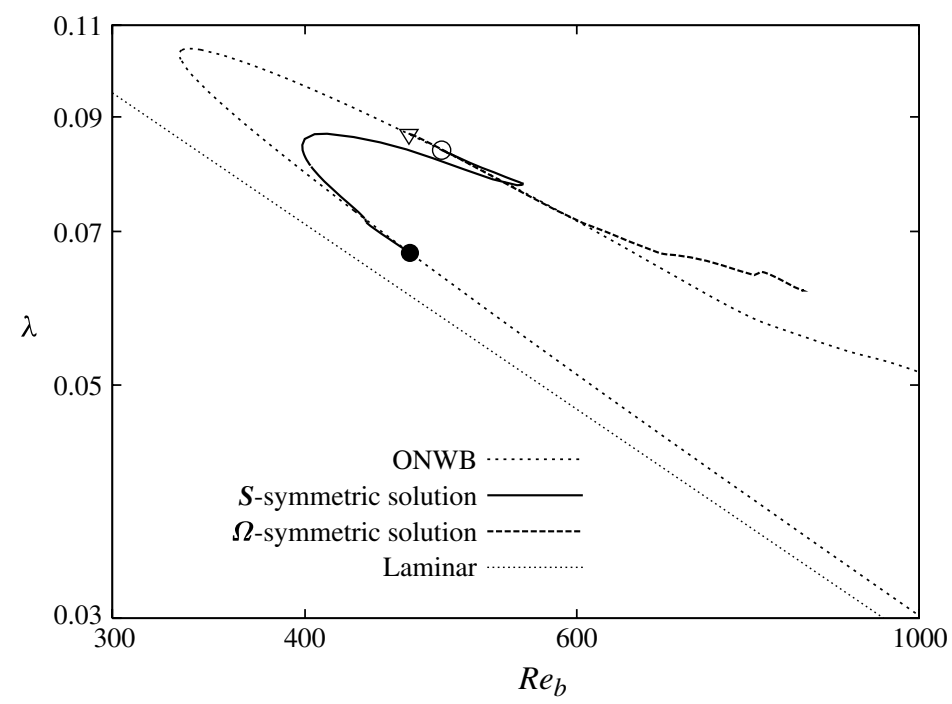

FIGURE 4. The skin friction against the bulk Reynolds number for $\alpha=1.14$. The curves and symbols are the same as figure 3 . The laminar state is given by $\lambda=28.45 / \operatorname{Re}_{b}$.

$\begin{array}{ccccc}(L, M, N) & c & R e_{b} & \lambda & \text { d.o.f. } \\ (6,33,33) & 2207.2 & 1342.0 & 0.02261 & 12483 \\ (7,35,35) & 2207.9 & 1341.5 & 0.02263 & 16323 \\ (8,37,37) & 2209.1 & 1341.4 & 0.02263 & 20811 \\ (9,39,39) & 2209.3 & 1341.6 & 0.02263 & 25995\end{array}$

TABLE 1. The truncation dependence of the phase velocity, the bulk Reynolds number, and the skin friction for the $S$-symmetric solution with $\alpha=0.70$ at $R e=3000$. The degrees of freedom are also shown.

$\begin{array}{ccccc}(L, M, N) & c & R e_{b} & \lambda & \text { d.o.f. } \\ (6,33,33) & 591.73 & 460.77 & 0.08951 & 12483 \\ (7,35,35) & 592.00 & 460.89 & 0.08946 & 16323 \\ (8,37,37) & 592.23 & 461.01 & 0.08942 & 20811 \\ (9,39,39) & 592.20 & 461.02 & 0.08941 & 25995\end{array}$

TABLE 2. As table 1 , but for the $\Omega$-symmetric solution with $\alpha=1.43$ at $R e=1400$.

levels $(L, M, N)=(6,33,33)$ for both the $S$-symmetric solution and the $\Omega$-symmetric solution after numerical convergence checks have been carried out (see tables 1 and 2). In the tables $L$ corresponds to the number of Fourier modes in the axial direction, and $M$ and $N$ correspond to the maximum degrees of the modified Chebyshev polynomials in the square cross-section. The degrees of freedom (d.o.f.) are almost doubled when compared with the 6242 used for ONWB. 

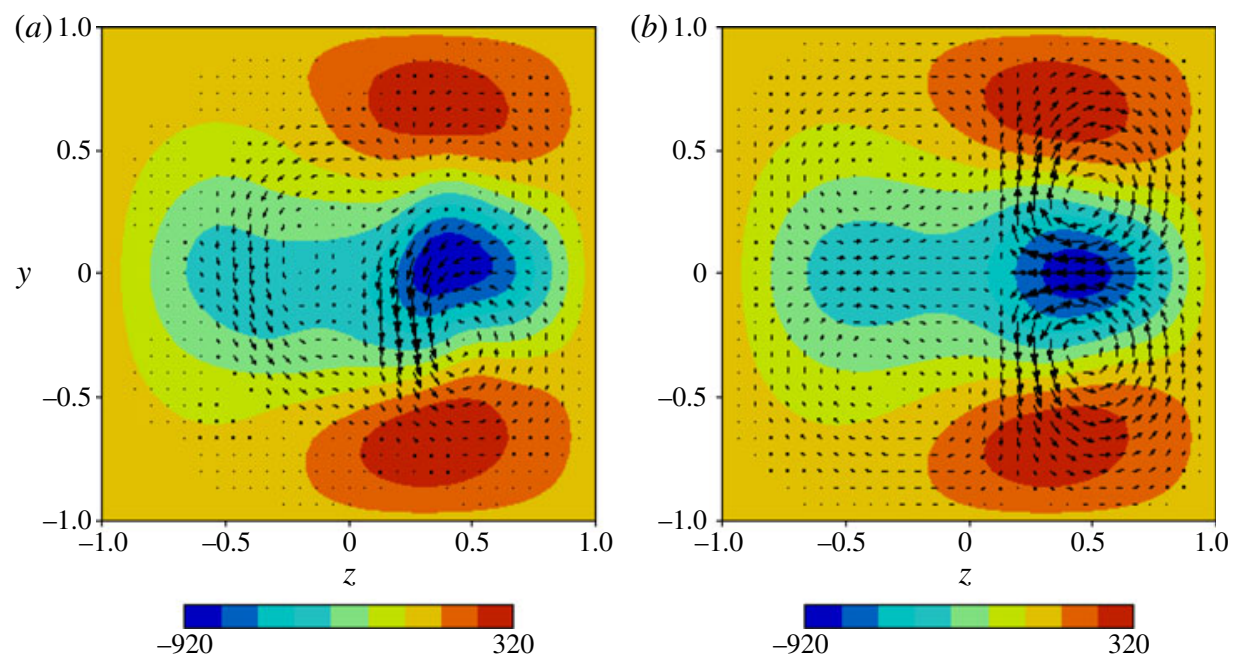

FIGURE 5. (a) The instantaneous flow and $(b)$ the mean part of the disturbance of the $S$-symmetric solution with $\alpha=0.5, R e=3315\left(R e_{b}=1468\right)$. The velocities on the $y-z$ plane and in the $x$-direction are indicated by the arrows and the colourscale, respectively.

\subsection{S-symmetric solution}

The instantaneous flow of the disturbance of the $S$-symmetric solution at $\xi \equiv x-c t=$ $5 \pi / 8$ with an appropriate choice of the initial phase and the mean part of the disturbance for $\alpha=0.5$ at $R e=3315$ are shown in figure 5(a) and figure 5(b), respectively. The mirror-symmetry with respect to $z=0$ of ONWB is broken and strong disturbances, which are composed of a pair of quasi-streamwise vortices and a low-speed streak, are observed near the sidewall $z=+1$. The solution which has the same structures near $z=-1$ is also a possibility by the symmetry of the system. The mean flow is mirror-symmetric with respect to $y=0$ (taking the streamwise average of (2.1) leads to the symmetry for the mean flow: $(\bar{U}, \bar{V}, \bar{W})(y, z)=(\bar{U},-\bar{V}, \bar{W})(-y, z)$, where $\bar{U}=\int_{0}^{2 \pi / \alpha} u \mathrm{~d} x$, etc.). The flow structures of this solution exhibit a striking similarity to those of the asymmetric solution in a pipe by Pringle \& Kerswell (2007) (see the top frames of their figure 1). These two asymmetric solutions possess the shift-and-reflect symmetry in common. Strong similarity for the two solutions is also observed in the skin friction (compare figure 4 with their figure 6). The existence domains of the $\boldsymbol{S}$-symmetric solution are shown in figure 6. We can see that for each of the three cases, $R e=950,1000,1050$, the lower and the upper branches of ONWB corresponding to the upper and lower parts of the closed loop are connected by the $S$-symmetric solution, indicated by a curve with the open and filled circles at its end points on the loop. The existence domain has more turning points on the $\alpha-R e_{b}$ plane as the Reynolds number increases, which indicates that the number of solutions increases for a fixed wavenumber. The $S$-symmetric travelling wave emerges first in the $\alpha-R e_{b}$ plane at $\alpha=1.36, R e=924\left(R e_{b}=374\right)$ and the minimum bulk Reynolds number is 365 at $\alpha=1.38, R e=952$.

The mean flow of our $\boldsymbol{S}$-symmetric solution resembles that of the edge state in the square-duct flow in Biau \& Bottaro (2009) (compare figure 5(b) with their figure 6). We also examine other flow properties of the $S$-symmetric solution to see whether the solution matches with the edge state identified in Biau \& Bottaro (2009), which 


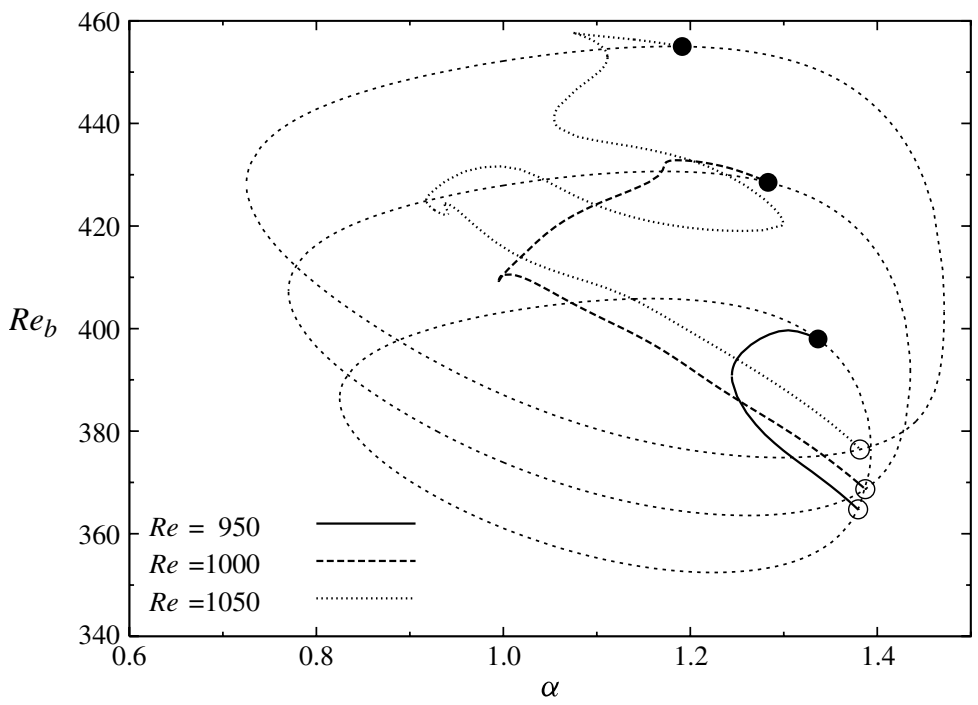

FIgURE 6. The existence domains of the $S$-symmetric solutions at $R e=950,1000,1050$. The dotted loops indicate ONWB. The filled (open) circles represent the bifurcation points on the lower (upper) branch.

\begin{tabular}{|c|c|c|c|}
\hline Solution & $\lambda$ & $R e_{b}$ & $E_{U}$ \\
\hline$S$-s & 02 & (2937) & 1.439 \\
\hline ONWE & 0.020 & 146 & $1.429 \times 10^{6}(254.1)$ \\
\hline WBN, lo & 0.03459 & 1145 (2290) & $8.172 \times 10^{5}(145.3)$ \\
\hline UKP, lower & 0.02568 & $1324(2648)$ & $1.120 \times 10^{6}(199.1)$ \\
\hline
\end{tabular}

TABLE 3. The skin friction $\lambda$, bulk Reynolds number $R e_{b}$ and energy of the streamwiseaveraged flow $E_{U}=(1 / 4) \int_{-1}^{+1} \int_{-1}^{+1}\left(\bar{U}^{2}+\bar{V}^{2}+\bar{W}^{2}\right) / 2 \mathrm{~d} y \mathrm{~d} z$ of the travelling waves with $R e=3315, \alpha=0.5$. The values in the brackets are normalized by the scales adopted in Biau \& Bottaro (2009). The solutions reported in Wedin et al. (2009) and Uhlmann et al. (2010) are referred to as WBN and UKP, respectively.

lies within $0.021 \lesssim \lambda \lesssim 0.024,2720 \lesssim R e_{b} \lesssim 2900$ and $214 \lesssim E_{U} \lesssim 250$, where $E_{U}$ is the energy of the streamwise-averaged flow (see their figures 5 and 7). The properties of the $S$-symmetric solution and the lower branches of other travelling wave solutions obtained elsewhere with $\alpha=0.5$ for $R e=3315$ (this parameter corresponds to $\alpha=1.0, R e_{\tau}=150$ in the notation of Biau \& Bottaro 2009) are listed in table 3 . From table 3 we see that the $S$-symmetric solution and ONWB take the values for $\lambda, R_{b}$ and $E_{U}$ almost within the ranges of the edge state, while WBN and UKP have rather higher friction and lower energy and bulk Reynolds number than the edge state. It is found that the $\boldsymbol{S}$-symmetric solutions have a fewer unstable eigenvalues and the values themselves are smaller than those of ONWB when linear stabilities of these flows are compared to all possible forms of perturbations (see table 4). The asymmetric structure and the few and low-valued positive eigenvalues associated with the $\boldsymbol{S}$-symmetric solution indicate that the $\boldsymbol{S}$-symmetric solution matches, more adequately than ONWB in phase space, with the quasi-stable edge state in 

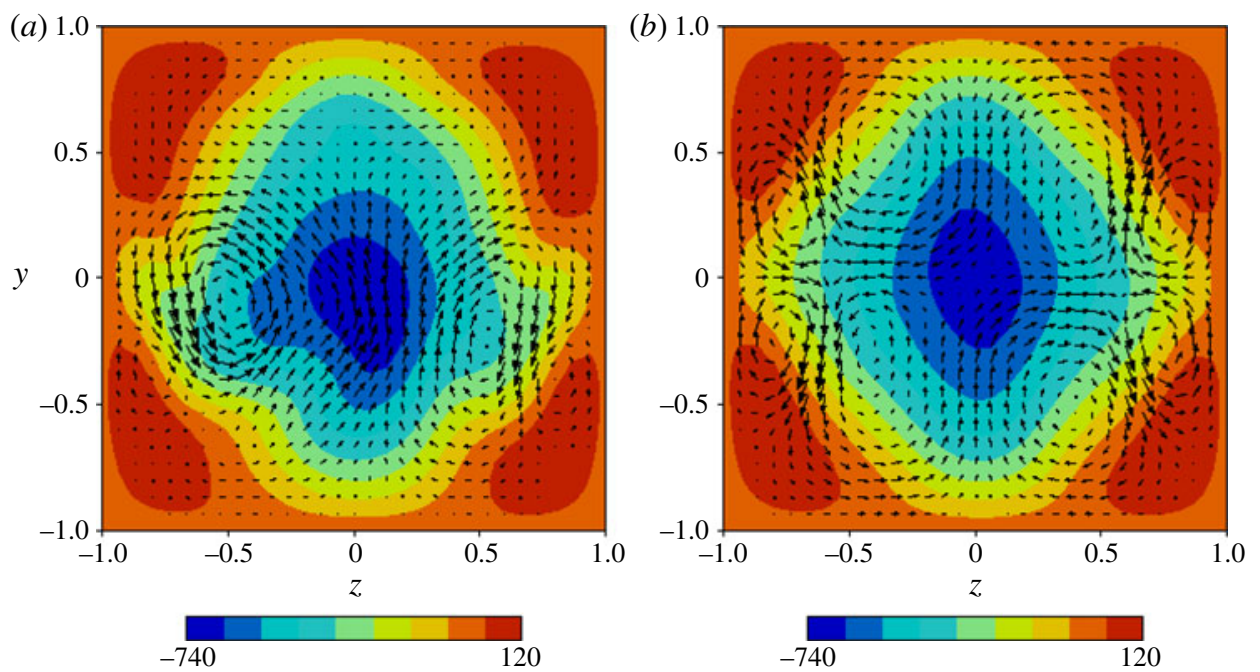

FIgURE 7. (a) The instantaneous flow and $(b)$ the mean part of the disturbance of the $\Omega$ symmetric solution with $\alpha=1.43, R e=1400\left(R e_{b}=461\right)$. The velocities on the $y-z$ plane and along the $x$-direction are indicated by the arrows and the colourscale, respectively.

$\begin{array}{lccccc}\text { Solution } & \sigma_{1} & \sigma_{2} & \sigma_{3} & \sigma_{4} & \sigma_{5} \\ \boldsymbol{S} \text {-symmetric } & 45.2 & 11.4 & 3.5 & 0 & -5.2 \\ \text { ONWB, lower } & 56.5 & 19.4 & 9.3 & 7.1 \pm 56.9 \mathrm{i} & 0\end{array}$

TABLE 4. The five largest eigenvalues of two travelling waves with $R e=3315, \alpha=0.5$.

Biau \& Bottaro (2009) where the vortical structure prefers one of the sidewalls and stays there for a while before moving to the other side. It can be expected that the edge state of square-duct flow is formed around the $\boldsymbol{S}$-symmetric solutions and their heteroclinic connections (four $S$-symmetric solutions exist due to the rotational symmetry by $90^{\circ}$ ) similar to those shown in Duguet et al. (2008b) for pipe flow. It should be noted that the edge state of pipe flow also consists of a pair of streamwise vortices located off centre (for instance, see Schneider et al. 2007).

\section{2. $\boldsymbol{\Omega}$-symmetric solution}

The velocity field of the $\boldsymbol{\Omega}$-symmetric solution is shown in figure 7 . The $\boldsymbol{\Omega}$-symmetric solution almost looks like the ONWB solution (see their figure 11), but the mirrorsymmetry $\boldsymbol{Z}$ with respect to $z=0$ is broken as can be seen in figure 7 . The bifurcation of this solution is of pitchfork type, producing a pair of solutions as shown in figure 8 . We can see in figure $8(a)$, for one of the two solutions, that the vortices with negative vorticity are elongated in the streamwise direction more than those with positive vorticity. Figure $8(b)$, for the other solution, shows an opposite structure. The existence domains of the $\boldsymbol{\Omega}$-symmetric solution are shown in figure 9 . The solution appears first at $\alpha=1.43, \operatorname{Re}=1271\left(\operatorname{Re}_{b}=427\right)$. At a slightly larger Reynolds number, 
66

\section{S. Okino and M. Nagata}
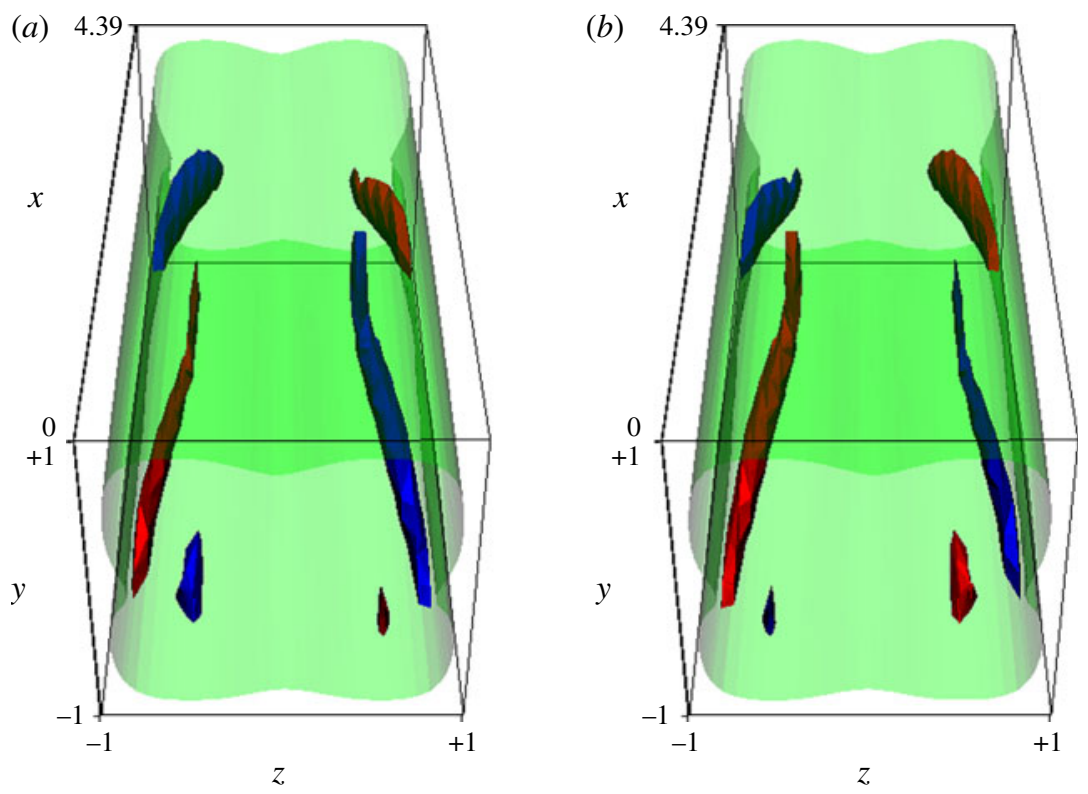

FIGURE 8. A pair of the $\Omega$-symmetric solutions with $\alpha=1.43, R e=1400\left(R e_{b}=461\right)$. The iso-surfaces, red (blue) and green, show $+70 \%(-70 \%)$ of the maximum streamwise vorticity and $40 \%$ of the maximum streamwise velocity, respectively.

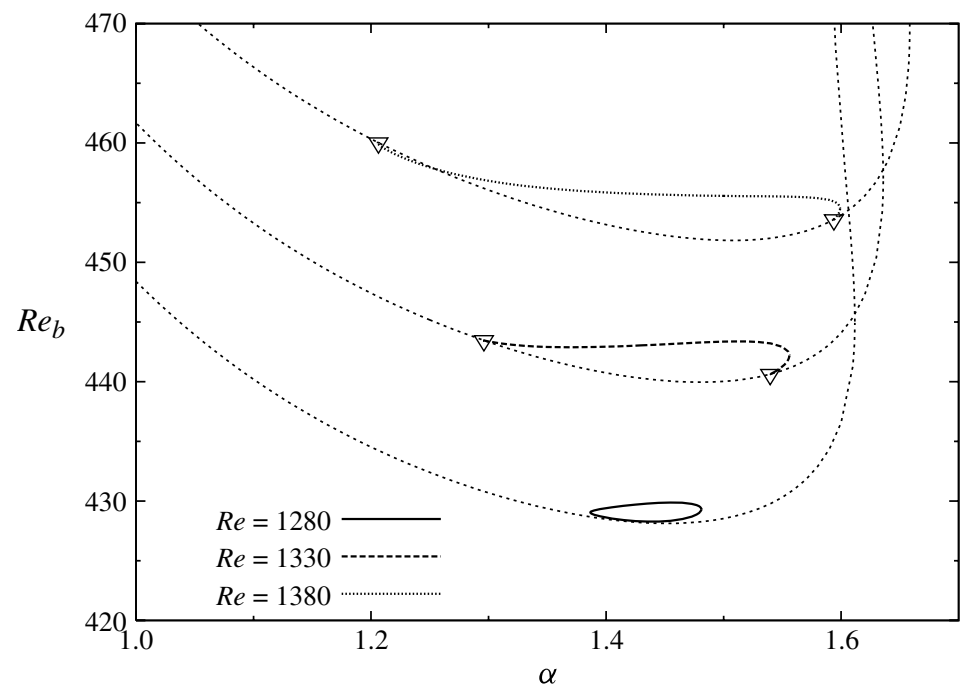

FIGURE 9. The existence domains of the $\Omega$-symmetric solutions at $R e=1280,1330,1380$.

The dotted curves indicate ONWB. The inverted triangles represent the bifurcation points.

the $\Omega$-symmetric solution begins to connect with the upper branch of ONWB via a pitchfork bifurcation (open inverted triangles). The counterpart of this solution in pipe flow has not been reported yet. 


\section{Conclusion}

We have performed a linear stability analysis of the mirror-symmetric solution obtained by Okino et al. (2010), and found the neutral points with a real eigenvalue. Two asymmetric travelling wave solutions, which emerge through a pitchfork bifurcation from the neutral points, are obtained. One of them, the $\boldsymbol{S}$-symmetric solution, retains the shift-and-reflect symmetry, and is characterized by a pair of streamwise vortices and a low-speed streak attached to one of the sidewalls. The solution seems to be embedded in the laminar-turbulent boundary found by Biau \& Bottaro (2009).

The similarity in bifurcation nature between square-duct flow and pipe flow shown in this paper implies that these two pressure-driven flows become turbulent through a common mechanism despite the difference between their cross-sectional geometries. The presence of the corners of the square duct does not seem to play a crucial role in transition to turbulence.

The other, $\boldsymbol{\Omega}$-symmetric solution, retains the shift-and-rotate symmetry. This solution deviates slightly from ONWB. It is plausible that the counterpart of the $\boldsymbol{\Omega}$-symmetric solution exists also in pipe flow.

Our stability analysis shows that many solutions could emerge far below the transitional Reynolds number, $R e_{b} \sim 1000$, via symmetry-breaking bifurcations (mainly from the upper branch), showing the richness of the nonlinear solutions and the complexity of phase-space dynamics in the transitional regime. Solutions which bifurcate from neutral points with the complex eigenvalue are not considered here. These solutions are expected to be time-periodic in the frame of reference moving with a constant speed and might be captured numerically by using a time-stepping code.

Okino (2011) investigated localized vortex structures by smoothly changing the aspect ratio, $A$. A single vortex state persisted for ONWB at least for $A<5$. Further investigations would be required to detect a snaking instability (Schneider, Gibson \& Burke 2010) for larger aspect ratios. Also, the asymmetric states of Itano \&Toh (2000) in plane Poiseuille flow could be recovered by our asymmetric travelling wave solutions for asymptotically large aspect ratios. A set of solutions other than those presented here could also be found in Okino (2011), where the solutions were obtained by the self-sustaining process method (Wedin et al. 2008).

\section{REFERENCES}

Biau, D. \& Bottaro, A. 2009 An optimal path to transition in a duct. Phil. Trans. R. Soc. Lond. A 367, 529-544.

Davey, A. \& Drazin, P. G. 1969 The stability of Poiseuille flow in a pipe. J. Fluid Mech. 36 (2), 209-218.

Duguet, Y., Pringle, C. C. T. \& Kerswell, R. R. 2008 a Relative periodic orbits in transitional pipe flow. Phys. Fluids 20, 114102.

Duguet, Y., Willis, A. P. \& Kerswell, R. R. $2008 b$ Transition in pipe flow: the saddle structure on the boundary of turbulence. J. Fluid Mech. 613, 255-274.

Faisst, H. \& Eckhardt, B. 2003 Travelling waves in pipe flow. Phys. Rev. Lett. 91, 224502.

ItAno, T. \& TOH, S. 2001 The dynamics of bursting process in wall turbulence. J. Phys. Soc. Japan 70, 703-716.

Mellibovsky, F. \& Meseguer, A. 2009 Critical threshold in pipe flow transition. Phil. Trans. R. Soc. Lond. A 367, 545-560.

OKINO, S. 2011 Nonlinear travelling wave solutions in square duct flow. PhD Thesis, Kyoto University. 
Okino, S., Nagata, M., Wedin, H. \& Bottaro, A. 2010 A new nonlinear vortex state in square-duct flow. J. Fluid Mech. 657, 413-429.

Pringle, C. C. T., Duguet, Y. \& Kerswell, R. R. 2009 Highly symmetric travelling waves in pipe flow. Phil. Trans. R. Soc. Lond. A 367, 457-472.

Pringle, C. C. T. \& Kerswell, R. R. 2007 Asymmetric, helical and mirror-symmetric travelling waves in pipe flow. Phys. Rev. Lett. 99, 074502.

SCHNEIDER, T. M. \& ECKHARDT, B. 2009 Edge states intermediate between laminar and turbulent dynamics in pipe flow. Phil. Trans. R. Soc. Lond. A 367, 577-587.

Schneider, T. M., Eckhardt, B. \& Yorke, J. A. 2007 Turbulence transition and the edge of chaos in pipe flow. Phys. Rev. Lett. 99, 034502.

SCHNEIDER, T. M., GiBson, J. F. \& BURKe, J. 2010 Snakes and ladders: localized solutions of plane Couette flow. Phys. Rev. Lett. 104, 104501.

Schneider, T. M., Gibson, J. F., Lagha, M., Lillo, F. D. \& Eckhardt, B. 2008 Laminar-turbulent boundary in plane Couette flow. Phys. Rev. E 78, 037301.

TAtsumi, T. \& Yoshimura, T. 1990 Stability of the laminar flow in a rectangular duct. J. Fluid Mech. 212, 437-449.

Uhlmann, M, Kawahara, G. \& Pinelli, A. 2010 Travelling-waves consistent with turbulence-driven secondary flow in a square duct. Phys. Fluids 22, 084102.

Uhlmann, M. \& NAGATA, M. 2006 Linear stability of flow in an internally heated rectangular duct. J. Fluid Mech. 551, 387-404.

Uhlmann, M., Pinelli, A., Kawahara, G. \& Sekimoto, A. 2007 Marginally turbulent flow in a square duct. J. Fluid Mech. 588, 153-162.

WALEFFE, F. 1997 Three-dimensional coherent states in plane shear flows. Phys. Fluids 9, 883-900.

Wedin, H., Biau, D., Bottaro, A. \& Nagata, M. 2008 Coherent flow states in a square duct. Phys. Fluids 20, 094105.

Wedin, H., Bottaro, A. \& Nagata, M. 2009 Three-dimensional travelling waves in a square duct. Phys. Rev. E 79, 065305.

Wedin, H. \& KeRSWELL, R. R. 2004 Exact coherent structures in pipe flow: travelling wave solutions. J. Fluid Mech. 508, 333-371. 